\title{
Gastric metaplasia and Campylobacter pylori infection of duodenum in patients with chronic renal failure
}

\author{
S SHOUSHA, C KEEN, R A PARKINS* \\ From the Departments of Histopathology and ${ }^{*}$ Gastroenterology, Charing Cross Hospital and Charing Cross \\ and Westminster Medical School, London
}

SUMMARY Duodenal biopsy specimens from 80 patients with chronic renal failure, who were undergoing haemodialysis, were examined by light microscopy for evidence of inflammation, gastric metaplasia, and Campylobacter pylori infection. Chronic duodenitis was present in $47(59 \%)$ of patients, of whom only seven $(\mathbf{9 \%})$ showed evidence of active inflammation. Gastric metaplasia was present in $50(62.5 \%)$ of patients, yet Campylobacter pylori was identified in only two patients $(2 \cdot 5 \%)$.

It is suggested that the duodenal environment of patients with chronic renal failure remains hostile to the growth of these organisms in spite of the presence of gastric metaplasia.

Recently the presence of Campylobacter pylori has been shown in areas of gastric metaplasia in the proximal parts of the duodenum in patients with nonspecific duodenitis and duodenal ulceration. ${ }^{1-3}$ The organisms are usually present within the mucus layer covering the mucosal surface. They do not invade the underlying tissue, yet acute inflammatory cells are usually present in these circumstances within the underlying mucosa and, occasionally, on the surface. It has been suggested that this acute inflammatory reaction is a response to the presence of $C$ pylori which can colonise the duodenal mucosal surface only in the presence of gastric metaplasia. ${ }^{3}$ Patients with chronic renal failure are reported to have a high incidence of gastric metaplasia, ${ }^{4}$ although they only rarely show an acute inflammatory reaction. ${ }^{5}$ We therefore thought that a study of the incidence of $C$ pylori colonisaton of the duodenum in these patients may be of interest, especially as regards the association between gastric metaplasia, $C$ pylori, and neutrophilic infiltration.

\section{Material and methods}

Duodenal biopsy specimens from 80 patients with chronic renal failure, who were referred to the gastroenterology clinic for routine endoscopic examination before renal transplantation, were studied. All patients were receiving hospital or home dialysis. No postoperative patients were included in this study.

Accepted for publication 16 November 1988
Upper gastrointestinal endoscopy was carried out using an Olympus GIF gastroscope. Between one and four (average $2 \cdot 2$ ) biopsy specimens were taken from the first and second parts of the duodenum. The specimens were immediately fixed in neutral formalin and later routinely processed for histological examination. Serial sections, varying between two and 34 (average 13 sections) were prepared from each biopsy specimen. Sections from all cases were stained with haematoxylin and eosin. Other sections from most cases were also stained with alcian blue/periodic acidSchiff for mucin, and a modified Giemsa ${ }^{6}$ or WarthinStarry stain for the demonstration of $C$ pylori. All sections were examined for evidence of chronic inflammation, acute inflammation, gastric metaplasia and $C$ pylori. The degree of inflammation and the extent of gastric metaplasia were graded into mild, moderate, and severe as described previously. ${ }^{7}$ A mild degree of gastric metaplasia referred to the presence of a single small focus of metaplastic cells usually at the tip of a villus, a moderate degree indicated the presence of multiple small foci or a large area of metaplasia occupying less than half the mucosal surface, while a severe degree referred to the presence of the change in more than half the biopsy surface.

The significance of the results was assessed by the fourfold tables of the $\chi^{2}$ test. Probabilities were calculated with one degree of freedom.

The duodenal ultrastructural appearance in 10 patients included in this study has been reported elsewhere. ${ }^{8}$ 
The duodenum in chronic renal failure

Table 1 Incidence of duodenitis, gastric metaplasia, and $C$ pylori colonisation of duodenum in patients with chronic renal failure undergoing haemodialysis

\begin{tabular}{lccc}
\hline & $\begin{array}{l}\text { Men } \\
(n=51)\end{array}$ & $\begin{array}{l}\text { Women } \\
(n=29)\end{array}$ & $\begin{array}{l}\text { Total } \\
(n=80)\end{array}$ \\
\hline Duodenitis: & $31(61 \%)$ & $16(55 \%)$ & $47(59 \%)$ \\
Chronic & 26 & 14 & 40 \\
Active chronic & 5 & 2 & 7 \\
Gastric metaplasia & $32(63 \%)$ & $18(62 \%)$ & $50(62.5 \%)$ \\
C pylori & $2(4 \%)$ & 0 & $2(2 \cdot 5 \%)$ \\
\hline
\end{tabular}

\section{Results}

Fifty one patients were male and 29 female. Their ages ranged between 15 and 96 years with a mean age of 45 years (table 1).

Evidence of inflammation was present in 47 cases $(59 \%)$. This was purely chronic, as indicated by an increase in mononuclear inflammatory cells in 40 cases, mild in 35 , and moderate in five cases. Acute inflammatory cells were seen in only seven cases $(9 \%)$. The degree of acute inflammation was mild in six and severe in one, and in all cases there was an associated increase in chronic inflammatory cells-that is, the inflammation was active chronic.

Gastric metaplasia was present in 50 cases $(62 \cdot 5 \%)$. Thirty one of these also had evidence of inflammation and included six of the seven cases with active chronic duodenitis as well as 25 cases with purely chronic inflammation. Eighteen cases had gastric metaplasia with no obvious increase in inflammatory cells (fig 1), and 16 cases had evidence of inflammation without metaplasia. The metaplasia was mild in 15 , moderate in 26 , and extensive in nine. The metaplasia in the six cases with active chronic inflammation was mild in one, moderate in four, and severe in one.

Metaplasia was present in 32 of the 51 men $(63 \%)$ and in 18 of the 29 women $(62 \%)$. The difference in incidence between men and women was not significant.

C pylori was identified by light microscopy in only two patients. Both were male and were 45 and 53 years old, respectively. The duodenum in both showed severe active chronic duodenitis with gastric metaplasia: the latter was moderate in one and extensive in the other. The affected villi were broadened and their lamina propria showed severe oedema and contained variable numbers of acute and chronic inflammatory cells (fig 2a). Large numbers of bacteria were seen on the duodenal surface in relation to areas of gastric metaplasia. The bacteria were easily identified in sections stained with haematoxylin and eosin as well as in the sections stained with the special stains (fig $2 b$ ). Thus the estimated incidence of $C$ pylori was two of 80 $(2.5 \%)$ of all cases, two of $50(4 \%)$ of cases with gastric metaplasia of any degree, two of $35(5.7 \%)$ of cases with moderate or severe metaplasia, two of seven $(29 \%)$ of all cases with active chronic duodenitis and two of six $(33 \%)$ of cases with active chronic duodenitis and gastric metaplasia.

\section{Discussion}

The results of this study confirm the high incidence of chronic duodenitis and gastric metaplasia of the duodenum in patients with chronic renal failure, which we have previously noted in a small series studied by electron microscopy. ${ }^{8}$ The $59 \%$ incidence of chronic duodenitis and $62.5 \%$ incidence of gastric metaplasia noted in this study was also comparable with the $61 \%$ and $75 \%$ incidence of duodenitis and metaplasia, respectively, reported by Franzin et al in a similar group of patients. ${ }^{4}$ Unlike these authors, however, who reported $30 \%$ incidence of gastric heterotopia in the duodenum of their patients, no similar lesions were seen in our cases.

The $9 \%$ incidence of active chronic duodenitis reported here is comparable with the $8 \%$ incidence reported by Margolis et al in a similar group of patients with renal failure, ${ }^{5}$ but much less than the $24 \%$ and $28 \%$ respective incidence previously reported by us ${ }^{7}$ and by others ${ }^{2}$ in patients with duodenal ulcer and non-ulcer dyspepsia and no renal disease. The incidence of active duodenitis in patients with renal failure was also less than the $11.7 \%$ incidence reported by Wyatt et al in patients with dyspepsia. ${ }^{3}$

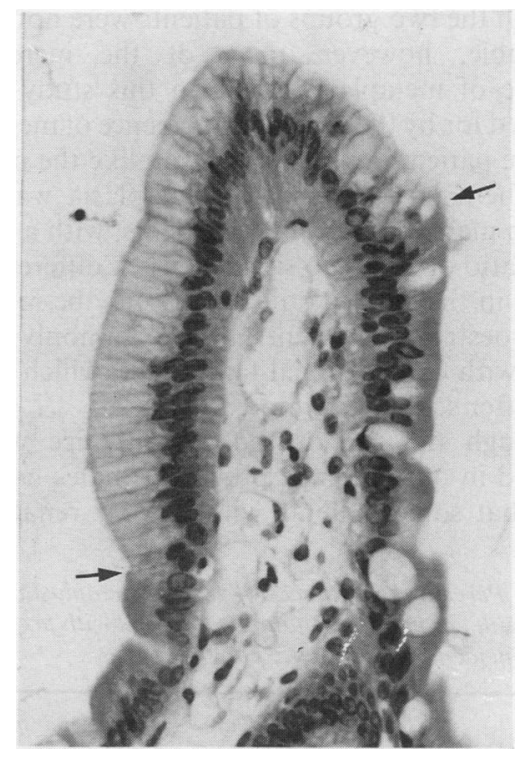

Fig 1 Duodenal villus showing gastric metaplasia (between arrows), but no surface colonisation with bacteria and no increase in inflammatory cells. 


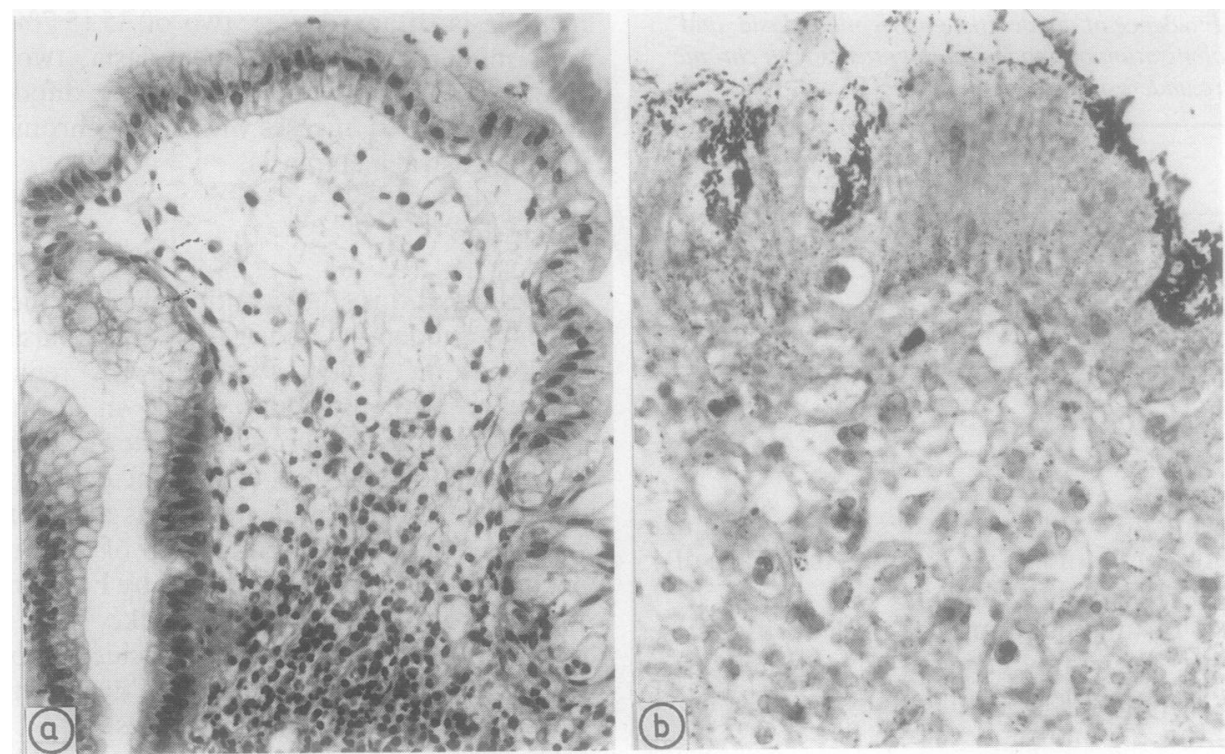

Fig 2 Duodenal villus showing surface gastric metaplasia and heavy colonisation with $C$ pylori organisms. (a) slide stained with haematoxylin and eosin showing broadening of villus, surface gastric metaplasia and oedema, and increased inflammatory cells of lamina propria. (b) Warthin-Starry stained section showing heavy colonisation of surface epithelium with $C$ pylori.

The $62.5 \%$ overall incidence of gastric metaplasia seen in this study was much higher than the $39 \%$ incidence seen by us in a previous study of 120 patients with non-ulcer dyspepsia and no known renal disease. ${ }^{9}$ Although the two groups of patients were not exactly comparable, however, most of the increase in incidence of metaplasia noted in this study can be accounted for by the increased incidence of metaplasia in female patients (table 2). Thus unlike the previous study where the incidence of metaplasia was much higher in male than in female patients, with a male to female ratio of $2 \cdot 6: 1$, no significant sex difference was detected in the current study. This may be related to the low oestrogen concentrations commonly seen in women with chronic renal failure and which tend to persist after starting haemodialysis. ${ }^{1011}$

Although the $\mathrm{pH}$ of the gastric juice was not measured in our patients, previous studies have suggested that some patients with chronic renal failure

Table 2 Percentage incidence of gastric metaplasia in 80 patients with chronic renal failure compared with previously. reported incidence in dyspeptic patients ${ }^{9}$

\begin{tabular}{lll}
\hline & Chronic renal failure & Non-ulcer dyspepsia \\
\hline Men & 63 & 54 \\
Women & 62 & 21 \\
Total & 62.5 & 39 \\
\hline
\end{tabular}

have increased gastric acidity. ${ }^{12}$ This may be another important factor connected with the presence of a high incidence of gastric metaplasia in these patients, as an association between high gastric acidity and duodenal gastric metaplasia has been previously noted clinically ${ }^{3}$ and experimentally. ${ }^{13}$

In spite of the high incidence of gastric metaplasia, the incidence of duodenal colonisation with $C$ pylori was unexpectedly low. Even if the cases showing mild metaplasia are excluded the incidence in those showing moderate and severe metaplasia remains at $5.7 \%$. This is much less than the $38 \%$ incidence reported by Wyatt et al in biopsy specimens from patients with dyspepsia showing comparable degrees of metaplasia (more than $5 \%$ of the epithelial surface). ${ }^{3}$

The low incidence of $C$ pylori in this study, however, was not entirely surprising as the incidence of histologically active duodenitis in our patients was low. A strong association has been previously shown between the presence of these organisms and active duodenitis, ${ }^{23}$ and our findings confirm this association. At the same time, these findings and those of Wyatt et al ${ }^{3}$ also suggest that active inflammation of the duodenum can occur in the absence of $C$ pylori and that this is more likely in patients with chronic renal failure: the incidence of these organisms in duodenal biopsy specimens showing active inflammatory and gastric metaplasia was $33 \%$ in our patients and $53 \%$ in patients with dyspepsia. ${ }^{3}$ 
It is not clear whether the low incidence of duodenal $C$ pylori, and probably consequent active inflammation, in spite of the presence of gastric metaplasia in our patients, is related to the renal failure itself or to accompanying therapeutic measures including haemodialysis.

Part of this study was presented, in an abstract form, at the first meeting of the European Campylobacter pylori study group, Bordeaux, France, October 1988.

\section{References}

1 Steer HW. Surface morphology of the gastroduodenal mucosa in duodenal ulceration. Gut 1984;25:1203-10.

2 Johnston BJ, Reed PI, Ali MH. Campylobacter like organisms in duodenal and antral endoscopic biopsies: relationship to inflammation. Gut 1986;27:1132-7.

3 Wyatt JI, Rathbone BJ, Dixon MF, Heatley RV. Campylobacter pyloridis and acid induced gastric metaplasia in the pathogenesis of duodenitis. J Clin Pathol 1987;40:841-8.

4 Franzin G, Musola R, Mencarelli R. Morphological changes of the gastroduodenal mucosa in regular dialysis uraemic patients. Histopathology 1982;6:429-37.

5 Margolis DM, Saylor JL, Geisse G, DeSchryver-Kecskemeti K, Harter HR, Zuckerman GR. Upper gastrointestinal disease in chronic renal failure. A prospective evaluation. Arch Intern Med
1978;138:1214-17.

6 Gray SF, Wyatt JI, Rathbone BJ. Simplified techniques for identifying Campylobacter pyloridis. J Clin Pathol 1986;39: 1279.

7 Shousha S, Spiller RC, Parkins RA. The endoscopically abnormal duodenum in patients with dyspepsia: biopsy findings in $\mathbf{6 0}$ cases. Histopathology 1983;7:23-34.

8 Shousha S, Bull TB, Parkins RA. Duodenal ultrastructure in patients with chronic renal failure. Ultrastruct Pathol 1989; (in press).

9 Shousha S, Barrison IG, El-Sayeed W, Khan S, Parkins RA. A study of incidence and relationship of intestinal metaplasia of gastric antrum and gastric metaplasia of duodenum in patients with non-ulcer dyspepsia. Dig Dis Sci 1984;29:311-16.

10 Feldman HA, Singer I. Endocrinology and metabolism in uremia and dialysis: A clinical review. Medicine 1974;54:345-76.

11 Brenner BM, Lazarus JM. Chronic renal failure: Pathophysiologic and clinical considerations. In: Braunwald $\mathrm{E}$, Isselbacher KJ, Petersdorf RG, Wilson JD, Martin JB, Fauci AS, eds. Harrison's principles of internal medicine. 11th ed. London; McGraw-Hill, 1987:1155-62.

12 Wesdorp RIC, Falcao HA, Banks PB, Martino J, Fischer JE. Gastrin and gastric acid secretion in renal failure. Am J Surg 1981;141:334-8.

13 Rhodes J. Experimental production of gastric epithelium in the duodenum. Gut 1964;5:454-8.

Requests for reprints to: Dr S Shousha, Department of Histopathology, Charing Cross Hospital, Fulham Palace Road, London W6 8RF, England. 